\title{
Muscle Spindle
}

National Cancer Institute

\section{Source}

National Cancer Institute. Muscle Spindle. NCI Thesaurus. Code C13824.

The Muscle Spindle is composed of intrafusal fibers that are located within and run parallel to the extrafusal fibers of skeletal muscle. When the extrafusal fibers are stretched, the intrafusal fibers of the spindle stretch as well. When stretching occurs, the sensory neuron from the muscle spindle signals the motor neurons located within the ventral horn of the spinal cord. This signal causes the motor neurons to fire, resulting in contraction of the muscle. This reflex arc provides negative feedback. The muscular contraction induced by the stretching works against or negates further muscle stretch. This mechanism helps to maintain proper muscle tension or tone. Although the muscle spindle helps to maintain proper muscle tension, unlike the Golgi tendon organ, it is not an indicator of muscle tension, but rather of muscle length. 